\title{
Herrn O. Univ.-Prof. Dipl.-Ing. Dr. Richard Eier zur Emeritierung
}

Im Jahr 1975, als ich Mitglied des Instituts für Datenverarbeitung an der Technischen Universität Wien wurde, war Prof. Dr. Eier Vorstand dieses neu gegrūndeten Instituts. Das Institut wuchs seither stetig, anstelle der ursprünglichen 2,5 Posten für Universitătsassistenten sind heute drei Professoren und 40 wissenschaftliche Mitarbeiter tätig. Es wurde zwischenzeitlich in Anbetracht seiner vielfältigen Tătigkeitsbereiche umbenannt und heißt nun Institut für Computertechnik.

Seine Wurzeln hat das Institut seit seiner Grūndung in jenen wissenschaftlichen Gebieten, in denen Prof. Eier selbst tätig war. Wesentliche Themen lagen dabei im Bereich der Informationstheorie, der Kryptografie, der Codierung, der stochastischen Prozesse und insbesondere der Markov-Prozesse. Das Institut fungierte damit als Brücke zwischen Implementierung und Anwendung der Computertechnik auf der einen Seite und den zugrunde liegenden Theorien auf der anderen Seite. Es war für die Mitarbeiter faszinierend zu erkennen, mit wie viel Routine und Effizienz Methoden der Matrizenrechnung ausgeführt werden können, und auch erschreckend, da klar war, dass man dem Institutsvorstand in diesem Bereich wohl wissenschaftlich nicht adăquat gegenübertreten konnte.

Schon kurz nach der Grūndung des Institutes entwickelte sich neben der Datenübertragung ein zweiter Themenschwerpunkt im Bereich der Mikroprozessoren. Neue Mitarbeiter, mich eingeschlossen, beschäftigten sich mit der Konzeption, Bewertung und der Anwendung der sich damals gerade sehr expansiv entwickelnden Mikroprozessortechnik. Die Industrie hatte in dieser frühen Phase der Anwendung von Mikroprozessoren einen sehr hohen Bedarf nach Experten, die sowohl grundlegende Forschungsarbeiten in diesem Bereich übernahmen als auch die Anwendung von Mikroprozessorsystemen unterstützen konnten. In der Zeit zwischen 1975 und 1980 ergaben sich aus der damaligen Pionierstellung des Instituts für Datenverarbeitung mannigfaltige und weitreichende Kontakte zu Industrieprojekten, Konsulententätigkeiten und Auftritten in den Medien, im Rahmen derer die Möglichkeiten und auch - damals heiß diskutiert - die Risken der Mikroprozessortechnik erlãutert wurden. Kaum eine Wissenschaftssendung, die sich mit diesem Thema in Funk oder Fernsehen auseinandergesetzt hat, konnte ohne fachmännischen Beitrag aus dem Institut für Datenverarbeitung auskommen.

Nach der Einfūhrung von Mikroprozessoren in der Datenverarbeitung war es nicht ungewöhnlich, dass die ersten industriellen Automatisierungssysteme auf universitären Entwicklungen fußten. Solche Entwicklungen aus dem Institut für Datenverarbeitung haben beispielsweise schon sehr früh Eingang in die Produktion von Coca Cola, von Margarine und in die Logistik von Großschlachthöfen, aber auch in die Verschlüsselung von Daten in militärischen Bereichen gefunden. Das Institut für Datenverarbeitung und die Arbeiten von Prof. Eier haben auf diesem Weg einen wesentlichen Beitrag dafūr geleistet, dass diese damals neuen Techniken in Österreich eine rasche Verbreitung gefunden haben. Österreichische Konzepte haben in weiterer Folge, beispielsweise in Form von multiprozessortähigen Bussystemen, europaweit Verbreitung gefunden. Die grundlegenden wissenschaftlichen Ansätze, die Prof. Eier aus dem Bereich

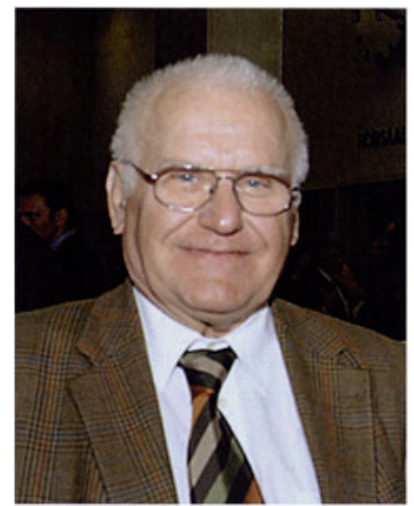

Emeritus Prof. Eier - Vollblutwissenschaftler, Vordenker, Vorbild für viele seiner Kollegen und Studenten. Stets offen zu raten und zu helfen

der Nachrichtentechnik in die damals neue Mikroprozessortechnik einbrachte, fūhrten schon zu einem sehr frühen Zeitpunkt zur Befassung mit zum Teil auch heute noch hochaktuellen Themen der Computerarchitektur. Beispiele dafür sind Konzepte zur Optimierung von Rechenalgorithmen, die Bewertung der „Máchtigkeit" von Befehlssätzen mit Hilfe der Entropiefunktion, die von Prof. Eier angeregt wurde, die Implementierung von redundanten Verfahren im Zuge der Speicherung und Verarbeitung von Daten in Mikroprozessoren und letztlich auch der praktische Einsatz der Kryptografie. Das methodische Hinterfragen der grundlegenden Technik hat es damals schon mit sich gebracht, dass das Institut auf Basis der Anregungen von Prof. Eier sehr frūh zur Entwicklung aktueller Konzepte, wie jener der RISC-Prozessoren, der methodischen Bewertung von Cachesystemen und Organisationskonzepten von Multiprozessorsystemen, maßgebliche Beitrăge geleistet hat. Diese Themen waren in den frühen siebziger Jahren allerdings keineswegs etabliert; damals begann sich die Mikroprozessortechnik gerade erst zu entwickeln. Anwendungsorientierte Konzepte wie Bussysteme, Multiprozessorsysteme und Methoden zur systematischen Applikationsentwicklung waren damals aktuelle Entwicklungen, die am Institut in zahlreichen Diplomarbeiten ihren Niederschlag gefunden haben.

Die wissenschaftlichen Erfolge und die Umsetzung dieser Konzepte in realen Produkten und Anwendungen sind nicht nur durch Arbeiten dokumentiert, die am Institut oder im Rahmen von Industriekooperationen entstanden sind, sondern manifestieren sich auch in den zahlreichen „Spin-offs“, die sich aus institutsaktivitāten ergeben haben. Als hervorragendes Beispiel dafür kann wohl die Firma Frequentis genannt werden, die im Bereich der Kommunikation in der Flugsicherungstechnik als Weltmarktführer agiert und dazu beiträgt, dass Spitzentechnologie und Arbeitsplātze in Österreich angesiedelt sind, wobei letztlich Konzepte und Verfahren, die am Institut für Computertechnik entwickelt wurden, den Ausgangspunkt für die heute angewandten Techniken darstellen. Im Schatten dieses Unternehmens befindet sich eine große Anzahl von Klein- und mittelständischen Untemehmen, Freiberuflern und Forschem, die in klei- 
nerem Rahmen, aber nicht minder erfolgreich, Konzepte und Erlerntes aus dem von Prof. Eier geleiteten Institut umsetzen. Die grundlegenden wissenschaftlichen Anregungen, das Einbringen mathematischer Methoden und das kritische Hinterfragen von Ergebnissen haben dabei für den Erfolg dieser Mitarbeiter ein wesentliches Element dargestellt. Das Institut war immer eine Forschungsstätte, in der die Verantwortung des Ingenieurs für seine Arbeit gelehrt und gelebt wurde. Die Einbeziehung von Kunst, Recht und Philosophie und die sich aus einem Selbstverständnis entwickelnde Ethik der Anwendung der Informationstechnologien waren für das Klima am Institut entscheidend. In einem solchen Institutsumfeld, in dem über jede Opernpremiere und über aktuelle philosophische Entwicklungen diskutiert werden konnte, und es selbstverständlich war, dass sich eine Fachdisziplin nur in einem humanistisch orientierten Umfeld entwickeln kann, war es leicht, leistungsfähige Ingenieure auszubilden und zu aktuellen Themen Beiträge bei Fernsehdiskussionen und in den Medien zu liefern.

Ein Geheimnis für den Erfolg, den Prof. Eier mit seinem Institut sowohl für die Technische Universităt Wien als auch für den Wirtschaftsstandort Österreich erzielen konnte, liegt aber nicht zuletzt in der Organisation und der Art der Mitarbeiterführung, die am Institut gepflogen wurde. Zurückblickend ergibt sich für mich heute, dass das Institut schon damals einem lebten“ Managementlehrbuch für Mitarbeiterführung im Sinne heute aktueller Methoden entsprach; diese nun selbstverständlichen Managementmethoden wurden damals einfach gelebt. Professor Eier hat immer versucht, die Stärken seiner Mitarbeiter zu finden und diese zu fordem. Daraus haben sich Fachgebiete entwickelt, in denen die Mitarbeiter nicht nur kompetent waren, sondern auch eine hohe Motivation fanden. Dabei hat sich Prof. Eier an die Tätigkeit seiner Mitarbeiter angepasst und es auf sich genommen, seinen wissenschaftlichen Beitrag jeweils so zu recherchieren und aufzubereiten, dass er für den jeweiligen Fachschwerpunkt wesentliche Anregungen und durchaus auch produktive Kritik einbringen konnte. Die Mitarbeiter konnten eigenstãndig arbeiten, hatten in ihrem Bereich eine hohe Entscheidungsbefugnis und das Wissen, über die Rückendeckung des Institutsvorstands zu verfügen, und auch bei Problemen jede erfordertiche Unterstützung zu erhalten. Aus einer solchen eigenständigen Tätigkeit entwickelt sich natürlich auch eine hohe Verantwortung für die eigenen Aktivitāten und Projekte, sowohl dem Institut und der Universităt als Arbeitgeber gegenüber als auch gegenüber dem technischen Fachgebiet selbst. Ein wesentliches Grundprinzip schien darin zu bestehen, Aktivităten zuzulassen und zu fördem und für Entscheidungen als Ratgeber zur Verfügung zu stehen. Diese Verantwortung hat auch dazu geführt, dass man gelernt hat, dass sämtliche Ergebnisse, bevor sie präsentiert oder publiziert wurden, besonders kritisch zu überprüfen waren. Man war verantwortlich, es musste einfach alles richtig sein. Ein Grundsatz, der meine weitere Tátigkeit - beispielsweise bei Gerichten - sehr erleichtert hat. Es galt bei einer Publikation nicht etwas zu schreiben, bei dem man keinen Fehler finden wird, sondern vor der Versendung jeden mōglichen Fehler noch selbst zu finden, ein Grundprinzip der Selbstkritik und Qualitătssicherung, das sich sehr bewährt hat. Wer Prof. Eier selbst dabei beobachten konnte, wie er seine eigenen Publikationen immer wieder kritisch verbessert hat, bis letztlich die unantastbare Version herausgegeben werden konnte, hat diese Hingabe an die Qualität verstanden und auch fūr den eigenen Bereich akzeptiert.

Die Mitarbeiter des Instituts hatten für ihre Bereiche nicht nur die Verantwortung, sie konnten auch sicher sein, den Erfolg genießen zu können. Für Prof. Eier war es nicht wichtig, bei den Arbeiten der Mitarbeiter genannt zu werden oder bei Prăsentationen in den Vordergrund zu treten, oder gar selbst die Ergebnisse der Mitarbeiter zu prăsentieren. Für inn war wichtig, dass die Mitarbeiter mit ihren Projekten selbst Erfolg hatten. Mit welcher Freude hat Prof. Eier einem nach einer erfolgreichen Prásentation oder Erwāhnung in den Medien gratuliertl Jeder hat dabei verstanden, dass der Erfolg der Schüler selbst und die Weiterentwicklung der Sache und des Instituts Prof. Eiers Erfolgsmaßstab war. Dass in einem solchen Umfeld guter Ton und Höflichkeit selbstverstăndlich waren, muss wohl nicht erwăhnt werden. Schon als junger Mitarbeiter hat man gelernt, dass sich auch der Institutsvorstand für zugeleistete Arbeiten bedankt und dass auch in kritischen Phasen Höflichkeit und Menschlichkeit das oberste Gebot zur Erreichung gemeinsamer Ziele sind.

Die Loyalität und die Unterstūtzung der Mitarbeiter war immer gesichert und auch selbstverståndlich. Konnte man eine Aufgabe, aus welchem Fachbereich auch immer, nicht lösen, war es selbstverständlich, dass sich der Institutsvorstand persönlich darum kümmerte. Meist führte dies nach einigen Tagen oder Nächten Arbeit zu einem Vorschlag für eine Lósung oder einen Lösungsweg. Unvergesslich sind mir jene Nächte, in denen uns Prof. Eier in Tătigkeitsbereichen, die üblicherweise Universitåtsprofessoren nicht zufallen, unterstūtzt hat. Selbst beim Bau von Prototypen und Geräten, die bei Tagungen pråsentiert werden mussten, hat er uns năchtelang persónlich geholfen. Allein durch diesen Einsatz hat er uns über manche schwierige Phasen hinweg zum Erfolg verholfen.

Professor Eier wechselt durch seine Emeritierung von einer Funktion in eine andere. Das ist ein Ereignis, das eine Rückschau auf seine erfolgreiche Schaffensperiode rechtfertigt. Prof. Eier hat seine Mitarbeiter erfolgreich motiviert und die von inm geliebte Technik und Wissenschaft weiter entwickelt. Das von inm geführte Institut hat Maßstäbe gesetzt, die Position Osterreichs im Bereich der Mikroprozessortechnik gestärkt und einen Ausgangspunkt und Katalysator für Firmen und Forschungsaktivitäten geschaffen, die weltweit erfolgreich agieren. Es ist ein Institut, in dem die technischen Wissenschaften in ein philosophisches und humanistisches Umfeld eingebettet waren, in dem die Mitarbeiter es gelernt haben, global zu denken und Verantwortung zu tragen. Das ist, nicht mehr und nicht weniger, ein Werk auf das Prof. Eier stolz sein kann.

Hon.-Prof. Dlpl.-Ing. Dr. Kurt P. Judmann OVE Judmann Ziviltechniker GmbH 